\title{
Cross Section Fluctuations of Photon Projectile in Generalized Vector Meson Dominance Model
}

\author{
L. Frankfurt \\ School of Physics and Astronomy, \\ Raymond and Beverly Sackler Faculty of Exact Sciences, \\ Tel Aviv University, 69978 Tel Aviv, Israel \\ and Inst. of Nuclear Physics, St. Petersburg, Russia \\ V. Guzey \\ Department of Physics \\ The Pennsylvania State University, University Park, PA 16802 \\ and \\ M. Strikman \\ Department of Physics \\ The Pennsylvania State University, University Park, PA 16802 \\ and Inst. of Nuclear Physics, St. Petersburg, Russia
}

\begin{abstract}
We explain that the generalized vector meson dominance (GVMD) model is successful in describing hard high energy processes because it contains expected in QCD Color Coherent Phenomena. Within the model, which reproduces Bjorken scaling and nuclear shadowing for deep inelastic processes, we

calculate $P_{\gamma}\left(\sigma, Q^{2}\right)$, the probability for the photon to interact with a target with the cross section $\sigma$. We find that within the GVMD model the virtual
\end{abstract}


photon has two main components: the "hard" one with the small cross section of the order of $1 / Q^{2}$ and the high probability and the "soft" one with a typical hadronic cross section with the probability suppressed by the factor of $1 / Q^{2}$. Thus the GVDM model produces $P_{\gamma}\left(\sigma, Q^{2}\right)$ similar to that within the parton model and suggests the conspiracy between hard and soft QCD at $Q^{2}>1 \mathrm{GeV}^{2}$.

We study the limit $\sigma \rightarrow 0$ and show, that $P_{\gamma}\left(\sigma, Q^{2}\right) \propto 1 / \sigma$ in both GVMD model and QCD. Thus similarly to the parton model the GVMD model predicts the Color Transparency phenomenon. This is an indirect indication that Bjorken scaling, Color Transparency and shadowing in nuclei in DIS are closely related.

\section{INTRODUCTION}

The aim of this paper is to demonstrate that a vector dominance model, generalized to comply with Bjorken scaling, (GVMD), contains Color Coherent Phenomena anticipated previously within the parton model and/or for hard PQCD regime. This explains the ability of GVDM to describe large sets of data and demonstrates once more that small $x$ physics of deep inelastic processes is a nontrivial interplay of soft and hard QCD physics.

Simple vector meson dominance (VMD) models, for review see [1], [2], [3] and references therein, describe successfully processes involving photon-hadron interactions at low "photon mass" $Q^{2}$. However such models, containing a finite number of vector mesons, do not reproduce approximate Bjorken scaling of $\sigma_{\gamma^{*} N}$ at large $Q^{2}$.

In order to restore the property of Bjorken scaling within GVDM, the photon was represented as an infinite sum of vector mesons [3]. The convergence of the sum within this model requires that the cross section of the meson-nucleon interaction decreases with the mass of the meson. In such models nuclear shadowing dies away as $Q^{2}$ increases, which contradicts recent experiments [4], which reveal only a weak dependence of $F_{2 A}\left(x, Q^{2}\right)$ on $Q^{2}$. 
This problem does not occur in generalized vector dominance (GVMD) models, where non-diagonal transitions among mesons have been introduced. The model we use conjectures that there are transitions only between mesons with neighboring masses [5], [6]. The scattering matrix, $\mathbf{S}$, then, acquires non-diagonal elements in the basis of vector mesons. The model describes reasonably well Bjorken scaling of $\sigma_{\gamma^{*} N}$ [5], [6] and nuclear shadowing in DIS [6].

Alternatively, one can expand the photonic state through states with the particular cross section of interaction with a target [7]. It is so called method of cross section fluctuations. The advantage of such an approach is that different states, having different cross section of interaction, do not mix, therefore the scattering matrix $\mathbf{S}$ or $\mathbf{T}$ is diagonal in such a basis.

The physical ground for this approach is the fact that configurations of a different geometrical size are present in the photon. It is known that the strength of the interaction of such a configuration depends on its geometrical size, consequently the photon can be represented as a superposition of eigenstates of the T-matrix, $\left|\psi_{n}\right\rangle$ :

$$
|\gamma\rangle=\sum c_{n}\left|\psi_{n}\right\rangle
$$

which interact with the target with different strengths:

$$
\mathbf{T}\left|\psi_{n}\right\rangle=\sigma_{n}\left|\psi_{n}\right\rangle
$$

It is convenient to introduce the distribution over cross sections $P_{\gamma}(\sigma)$, which gives the probability for the photon to interact with the target with the cross section $\sigma$. Having found the set of eigenvalues $\sigma_{n}$ and corresponding coefficients $c_{n}$, one can reconstruct the distribution $P_{\gamma}(\sigma)$ according to the rule []], [8]:

$$
P_{\gamma}(\sigma)=\sum_{n}\left|c_{n}\right|^{2} \delta\left(\sigma-\sigma_{n}\right)
$$

There are no methods allowing for a calculation of the distribution $P_{\gamma}\left(\sigma, Q^{2}\right)$ from the first principles, except for $\sigma \rightarrow 0$. In the present paper we evaluate $P_{\gamma}\left(\sigma, Q^{2}\right)$ within the framework of the GVMD model and find conspiracy between hard and soft physics suggested 
initially within the parton model [13]: although the effective cross section decreases as $1 / Q^{2}$, the probability of configurations interacting with a typical hadronic cross section is $1 / Q^{2}$ also. Thus the significant contribution of non-diagonal transitions resolves the Gribov puzzle [9]-the contradiction of pre-QCD ideas to approximate Bjorken scaling for deep inelastic processes.

We study asymptotic properties of $P_{\gamma}\left(\sigma, Q^{2}\right)$ at small $\sigma$ and compare predictions obtained in the GVMD model and QCD.

Once again we want to point out that the very fact of existence of the distribution over

cross sections $P_{\gamma}\left(\sigma, Q^{2}\right)$ proves that there are configurations of different strengths in the photon.

\section{EVALUATION OF $P_{\gamma}\left(\sigma, Q^{2}\right)$ WITHIN GVMD MODEL}

At high energies a photon interacts with a target by means of its hadronic components. The generalized vector meson dominance model that we use [5], [6] can be expressed as the following decomposition:

$$
|\gamma\rangle=\sum_{n}^{\infty} \frac{e}{f_{n}} \frac{M_{n}^{2}}{M_{n}^{2}+Q^{2}}\left|\rho_{n}\right\rangle .
$$

Parameters of the model are chosen such that

$$
M_{n}^{2}=M_{0}^{2}(1+2 n),
$$

where $M_{0}=0.77 \mathrm{GeV}$, assuming $\left|\rho_{0}\right\rangle=|\rho\rangle$, and

$$
\frac{M_{n}^{2}}{f_{n}^{2}}=\frac{M_{0}^{2}}{f_{0}^{2}},
$$

where $f_{0} / 4 \pi=2.36$.

Note that for simplicity we consider only one kind of vector mesons here, discarding contributions of $\omega$ and $\phi$ mesons. The inclusion of additional flavors would not change qualitative conclusions of this paper.

The total photoabsorption cross section for transverse photons on nucleons is [5], [6]: 


$$
\sigma_{T}\left(s, Q^{2}\right)=\sum_{n m} \frac{e}{f_{n}} \frac{M_{n}^{2}}{M_{n}^{2}+Q^{2}} \Sigma_{n m} \frac{e}{f_{m}} \frac{M_{m}^{2}}{M_{m}^{2}+Q^{2}} .
$$

$\Sigma_{n m}$ is the scattering matrix in the basis of vector mesons. Assuming that there are transitions only between the mesons with neighboring masses, this matrix takes up a symmetrical tridiagonal form [5], [6] :

$$
\Sigma_{n m}=\sigma_{0} \delta_{n m}-\frac{\sigma_{0}}{2} \frac{M_{n}}{M_{n+1}}\left(1-0.3 \frac{M_{0}^{2}}{M_{n}^{2}}\right) \delta_{n m \pm 1},
$$

where $\sigma_{0}=25 \mathrm{mb}$ is the total cross section of interaction of vector mesons with the target.

Non-diagonal elements of the matrix $\Sigma_{n m}$ are chosen in such a way that at large masses $M_{n}$ their first terms cancel diagonal elements. Their second terms have the form of a simple (nongeneralized) vector meson dominance model with the cross section inversely proportional to the mass squared of the mesons. Thus, the model [5], [6] reproduces approximate Bjorken scaling at relatively large $Q^{2}$ in spite of the fact that the cross section of the diagonal transitions does not decrease with $M_{n}^{2}$.

We want to stress that within conventional approaches such as the parton model, PQCD, nonrelativistic quark models of a hadron, cross sections of the scattering of excited hadronic states off a hadron target are not very different (may be larger) than the cross section of the ground state off the same target.

The scattering matrix $\mathbf{T}$ is diagonal in the basis of eigenvectors $\left|\psi_{n}\right\rangle$ with eigenvalues $\sigma_{n}$ giving possible cross sections. Thus, the problem of relating the two formalisms reduces to finding eigenvalues and eigenvectors of $\Sigma_{n m}$. Having found the representation of the vector of state of each meson in the basis of eigenvectors, and using (파), one can find coefficients $c_{n}$ in decomposition (11) and, thus, reconstruct $P_{\gamma}\left(\sigma, Q^{2}\right)$ according to (3).

The numerical solution to the problem was carried out with help of the computer software Mathematica for 10 mesons. The graphic solution is given in Fig. 1 for $Q^{2}=0,1,2 \mathrm{GeV}^{2}$ (we plot $\left.f_{0}^{2} / e^{2} \cdot P\left(\sigma, Q^{2}\right)\right)$. For such $Q^{2}$ it is sufficient to take into account only first ten mesons.

A more universal characteristic, which depends weakly on a number of vector mesons, is moments of the distribution $P_{\gamma}\left(\sigma, Q^{2}\right)$ defined as: 


$$
\left\langle\sigma^{n}\right\rangle=\int P_{\gamma}\left(\sigma, Q^{2}\right) \sigma^{n} d \sigma
$$

We give first five moments computed for our specific example of 10 vector mesons in Table (II), although the moments are not sensitive to the number of mesons taken into consideration.

From Fig. 1 one can see the following general tendencies of behavior of $P_{\gamma}\left(\sigma, Q^{2}\right)$ :

1. $P_{\gamma}\left(\sigma, Q^{2}\right) \propto 1 / \sigma$ at small $\sigma$

2. $P_{\gamma}\left(\sigma, Q^{2}\right) \rightarrow 0$ at large $\sigma$

3. $P_{\gamma}\left(\sigma, Q^{2}\right)$ decreases on average with increase of $\sigma$

4. $\left\langle\sigma^{n}\right\rangle \propto 1 / Q^{2}$ for $\mathrm{n}=1,2 \ldots$

Generally speaking, though the exact analytical form of the distribution $P_{\gamma}\left(\sigma, Q^{2}\right)$ depends on a number of vector mesons taken into account ( on the dimension of $\Sigma_{n m}$ ), the general tendencies given above reflect universal properties of the distribution $P_{\gamma}\left(\sigma, Q^{2}\right)$.

For calculation purposes it is useful to have an analytical expression of $P_{\gamma}\left(\sigma, Q^{2}\right)$ defined for all $\sigma$. We suggest the following parameterization, which reflects the general properties of $P_{\gamma}\left(\sigma, Q^{2}\right)$ :

$$
\begin{aligned}
P_{\gamma}\left(\sigma, Q^{2}\right) & =N\left(\frac{1}{\sigma / \sigma_{0}} \Theta\left(\frac{1}{C} \frac{\mu_{1}^{2}}{\mu_{1}^{2}+Q^{2}}-\sigma / \sigma_{0}\right)+\frac{\mu^{2}}{\mu^{2}+Q^{2}} \Pi\left(\sigma / \sigma_{0}\right)\right) \\
\Pi\left(\sigma / \sigma_{0}\right) & =6.03 \exp \left(-15.15 \cdot\left(\sigma / \sigma_{0}-0.7\right)^{2}\right) \\
\mu_{1}^{2} & =0.32 \mathrm{GeV}^{2} \\
\mu^{2} & =0.39 \mathrm{GeV}^{2} \\
C & =0.589 \\
N & =0.387
\end{aligned}
$$

Here $\Theta$ is a step-function. The presented parameterization reproduces first three moments of the actual distribution $P_{\gamma}\left(\sigma, Q^{2}\right)$ quite accurately for $0<Q^{2}<1 \mathrm{GeV}^{2}$ : the total cross section is given with the accuracy of $4 \%$, the second moment is fitted with the accuracy of $8 \%$, the third moment accuracy is at the level of $15 \%$. 
One can see that this distribution contains a nontrivial interplay between hard and soft physics. The first term is a "hard" piece with the cross section proportional to $1 / Q^{2}$ and the probability which does not depend on $Q^{2}$. The second term is a "soft" piece with the large cross section and the probability which dies away as $1 / Q^{2}$. While both terms contribute to the total cross section, the dominant contribution to higher moments comes from the soft part of $P_{\gamma}\left(\sigma, Q^{2}\right)$ which guarantees that nuclear shadowing is the leading twist effect. One can also see it from discrete versions of the distribution $P_{\gamma}\left(\sigma, Q^{2}\right)$ for 10 and 20 vector mesons.

We have shown that this particular GVMD model leads to the existence of the nontrivial distribution over cross section, which agrees with the notion that the photon consists of $q \bar{q}$ configurations, having different geometrical sizes, therefore interacting with different cross sections. This phenomenon is called cross section fluctuations.

\section{NUCLEAR SHADOWING}

In the total cross section of deep inelastic $\gamma^{*} A$ scattering nuclear shadowing is predominantly inelastic shadowing, that is due to high mass intermediate states [9]. The leading contribution (double rescattering) can be expressed in terms of the cross section of inclusive forward diffractive dissociation of the virtual photon into states " $X^{\prime}: \gamma^{*}+N \rightarrow X+N$.

The natural assumption, that any hadron state interacts with sufficiently heavy nuclei with the same cross section $\pi R_{A}^{2}$, leads to the Gribov relationship between the total photoabsorption cross section and cross section of the process $e \bar{e} \rightarrow$ hadrons, which contradicts Bjorken scaling [12]. The idea how to resolve the Gribov puzzle has been suggested by Bjorken [13]. He applied the parton model to the light cone wave function of the energetic photon and concluded that the momentum of the quark (antiquark) in the photon should be aligned along the momentum of the photon. Such rare,- with the probability $1 / Q^{2},-$ large size, asymmetric configurations give the dominant contribution into the cross section -the

aligned jet model [13]. Thus most of quark-antiquark configurations in the photon are sterile 
within the parton model approximation.

In QCD this picture is modified by identifying sterile states as colorless quark-gluon configurations of a spatially small size having small interaction cross sections and by including QCD evolution of sterile states. QCD evolution leads to a fast increase with energy of the cross section of interaction of quark configurations having large $k_{t}$ and to the parton bremsstrahlung [14]. Thus the QCD improved aligned jet model predicts a nontrivial competition between two contributions into $P_{\gamma}\left(\sigma, Q^{2}\right)$. The soft piece corresponds to a usual hadronic cross section but with the probability $1 / Q^{2}$. The hard piece to $P_{\gamma}\left(\sigma, Q^{2}\right)$ comes from cross sections $\sigma \propto 1 / Q^{2}$, but their probability does not decrease with $Q^{2}$.

Another useful representation of the cross section of inclusive forward diffractive dissociation of the virtual photon into states " $X$ " and deep inelastic $\gamma^{*} A$ scattering can be given in terms of cross section fluctuations [10]. Within this representation nuclear shadowing in DIS is a consequence of significant cross section fluctuations. Nuclear shadowing in DIS is given by the series of terms such as $\sigma^{n}$. The first term in this series is $\propto\left\langle\sigma^{2}\right\rangle /\langle\sigma\rangle$ [10], [11].

The diagonal vector meson dominance model assumes that cross section of the interaction of hadronic components of the photon falls off as the inverse mass squared of the component, $\sigma_{h} \propto 1 / m_{h}^{2}$. So within this model $\langle\sigma\rangle \propto 1 / Q^{2},\left\langle\sigma^{2}\right\rangle \propto \ln \left(Q^{2}\right) / Q^{4}$, which makes

$$
\frac{\left\langle\sigma^{2}\right\rangle}{\langle\sigma\rangle}=\frac{\ln \left(Q^{2}\right)}{Q^{2}}
$$

Therefore within this model nuclear shadowing dies out at large $Q^{2}$ as a power of $1 / Q^{2}$ which contradicts the experimental data.

The generalized vector meson dominance model that we use resembles the aligned jet model in the sense that the meson-nucleon cross section does not depend on the mass of the meson, which preserves the scaling of shadowing. The cancelation of diagonal terms by off-diagonal ones brings the additional factor of $1 / m_{h}^{2}$ which restores the Bjorken scaling. Therefore, these two properties exhibited by the GVMD model are possible only when the photon has both "hard" and "soft" components. The ability of the GMVD model to describe the experimental data stems from the fact the model takes into account basic coherent QCD 
phenomena such as cross section fluctuations, which arise due to non-diagonal transitions, and the Color Transparency Phenomenon, which was taken into account in the particular choice of non-diagonal terms.

Using the exact form of $\Sigma_{n m}$ (8) one can present with good accuracy the total photoabsorption cross section at $Q^{2}>1 \mathrm{GeV}^{2}$ :

$$
\sigma_{T}\left(s, Q^{2}>1 G e V^{2}\right)=\frac{e^{2}}{f_{0}^{2}} \sigma_{0} \sum_{n} \frac{M_{0}^{4}}{\left(M_{n}^{2}+Q^{2}\right)^{2}}\left(\frac{M_{n}^{2}}{M_{n}^{2}+Q^{2}}+0.3\right),
$$

which exhibits $1 / Q^{2}$ behavior.

Using the formalism of cross section fluctuations, one can show that the second moment of the cross section in the adopted model is given as

$$
\left\langle\sigma^{2}\left(s, Q^{2}\right)\right\rangle=\sum_{n m k} \frac{e}{f_{n}} \frac{M_{n}^{2}}{M_{n}^{2}+Q^{2}} \Sigma_{n k} \Sigma_{k m} \frac{e}{f_{m}} \frac{M_{m}^{2}}{M_{m}^{2}+Q^{2}} .
$$

It turns out that $\Sigma_{n k} \Sigma_{k m} \propto 1 / M_{n}^{2}$ for large $n, m, k$, which leads to

$$
\left\langle\sigma^{2}\left(s, Q^{2}\right)\right\rangle \propto \frac{1}{Q^{2}}
$$

for $Q^{2}>1 \mathrm{GeV}^{2}$.

Numerical calculations were carried out for 10 vector mesons. The dependence of $\left\langle\sigma^{2}\left(s, Q^{2}\right)\right\rangle /\left\langle\sigma\left(s, Q^{2}\right)\right\rangle$ on $Q^{2}$ is presented in Fig. 2, which shows that for $Q^{2}>5 \mathrm{GeV}^{2}$ shadowing becomes independent on $Q^{2}$. Note that since our aim in this paper is to investigate soft QCD physics, we ignore QCD evolution due to hard radiation. At comparison with the experimental data QCD evolution should be taken into account.

\section{ASYMPTOTIC BEHAVIOR OF $P_{\gamma}\left(\sigma, Q^{2}\right)$ AT SMALL $\sigma$}

Using the explicit form of $\Sigma_{n m}$ given by Eq. (8), one can write the total photoabsorption cross section (17):

$$
\sigma_{T}\left(s, Q^{2}\right)=\sum_{n} \frac{e}{f_{n}} \frac{M_{n}^{2}}{M_{n}^{2}+Q^{2}}\left(\sigma_{0} \frac{e}{f_{n}} \frac{M_{n}^{2}}{M_{n}^{2}+Q^{2}}+2 c_{n} \frac{e}{f_{n+1}} \frac{M_{n+1}^{2}}{M_{n+1}^{2}+Q^{2}}\right),
$$

where 


$$
c_{n}=-\frac{\sigma_{0}}{2} \frac{M_{n}}{M_{n+1}}\left(1-0.3 \frac{M_{0}}{M_{n+1}}\right) .
$$

Numerical studies show that if one wants to study the limit of small $\sigma$, one should increase the number of vector mesons included in sum (15) (the dimensionality of $\Sigma_{n m}$ ). As a result the eigenvalues (possible cross sections) $\sigma_{n}$ fill the interval $\left[0,2 \sigma_{0}\right]$ more and more densely. Therefore, the minimal eigenvalue (cross section) decreases with increase of the number of vector mesons $L$. Note that when $L$ is a large number, $\frac{M_{0}^{2}}{M_{L}^{2}+Q^{2}}$ is a small parameter, hence the expression for $\sigma_{T}\left(s, Q^{2}\right)$ can be simplified further:

$$
\sigma_{T}\left(s, Q^{2}\right)=2 e^{2} \frac{M_{0}^{2}}{f_{0}^{2}} \sum_{L} \frac{M_{L}^{2}}{\left(M_{L}^{2}+Q^{2}\right)^{2}} \sigma_{0}\left(0.15 \frac{M_{0}^{2}}{M_{L}^{2}}+\frac{M_{0}^{2}}{M_{L}^{2}+Q^{2}}\right) .
$$

The ultimate goal of this subsection is to express $\sigma_{T}\left(s, Q^{2}\right)$ as an integral over cross sections. We have explained that large masses $M_{L}^{2}$ correspond to small cross sections, therefore we use the following fit for the calculated minimal cross section (eigenvalue of $\Sigma_{n m}$ ) for large $M_{L}^{2}(\operatorname{large} L):$

$$
\sigma\left(M_{L}^{2}\right)=A \sigma_{0} \frac{M_{0}^{2}}{M_{L}^{2}}
$$

Numerical works with $\Sigma_{n m}$ reveal that $A$ does not depend significantly on $N$ for its large enough values, for example, for $N=85 A=2.12$, for $N=100 A=2.06$, for $N=200$ $A=1.88$. In our numerical analysis we use the averaged value $A=2$.

Since for large masses (large $L$ ) the relative difference between neighboring cross sections $\sigma\left(M_{L}^{2}\right)$ is small, it is accurate to replace this sum by an integral over cross sections $\sigma$ (keeping in mind that we integrate over small cross sections):

$$
\sigma_{T}\left(s, Q^{2}\right)=\int d \sigma \frac{e^{2}}{f_{0}^{2}} \sigma_{0} \frac{1}{\left(\sigma / \sigma_{0}\right)^{2}} \frac{A M_{0}^{4}}{\left(A M_{0}^{2} \sigma_{0} / \sigma+Q^{2}\right)^{2}} \cdot\left(0.15+\frac{1}{\sigma / \sigma_{0}} \frac{A M_{0}^{2}}{A M_{0}^{2} \sigma_{0} / \sigma+Q^{2}}\right) .
$$

According to the definition of $P_{\gamma}\left(\sigma, Q^{2}\right)$ :

$$
\sigma_{T}\left(s, Q^{2}\right)=\int d \sigma P_{\gamma}(\sigma) \sigma
$$

Comparing these two expressions, we notice that that at small $\sigma$ : 


$$
P_{\gamma}\left(\sigma, Q^{2}\right)=\frac{e^{2}}{f_{0}^{2}} \frac{1}{\left(\sigma / \sigma_{0}\right)^{3}} \frac{A M_{0}^{4}}{\left(A M_{0}^{2} \sigma_{0} / \sigma+Q^{2}\right)^{2}} \cdot\left(0.15+\frac{1}{\sigma / \sigma_{0}} \frac{A M_{0}^{2}}{A M_{0}^{2} \sigma_{0} / \sigma+Q^{2}}\right) .
$$

One can see that this formula supports our statement that $P_{\gamma}\left(\sigma, Q^{2}\right) \propto 1 / \sigma$ at small $\sigma$. It shows that small cross sections have a sizable probability to exist in the photon. This phenomenon leads to Color Transparency Phenomena.

To make numerical estimate of the coefficient of proportionality, we present the distribution $P_{\gamma}\left(\sigma, Q^{2}\right)$ as

$$
P_{\gamma}\left(\sigma, Q^{2}\right)=e^{2} \frac{1}{\sigma} I_{V M D}\left(Q^{2}\right),
$$

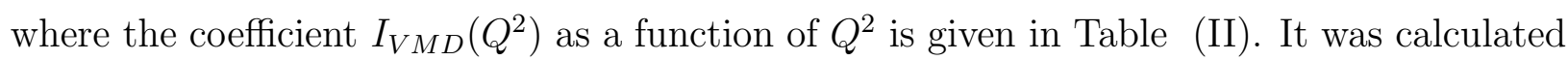
for $\sigma / \sigma_{0}=0.1$.

There is an alternative approach to the calculation of the asymptotic (limiting) behavior of $P_{\gamma}\left(\sigma, Q^{2}\right)$ based on QCD, which presents the answer in terms of the photon wave function and the cross section of interaction of a small size $q \bar{q}$-configuration [15]:

$$
P\left(\sigma, Q^{2}\right)=\frac{d b^{2}}{d \sigma\left(b^{2}\right)} \int_{0}^{1} \sum_{\lambda_{1}, \lambda_{2}}\left|\psi(z, b=0)_{\lambda_{1}, \lambda_{2}}\right|^{2} \frac{d z}{4} N_{C} .
$$

Here $\psi(z, \vec{b})_{\lambda_{1}, \lambda_{2}}$ is a light-cone wave function of the photon made of two quarks with momentum fractions $z$ and $1-z$ and helicities $\lambda_{1}$ and $\lambda_{2} ; \sigma\left(b^{2}\right)$ is the cross section of the $q \bar{q}$ pair of the transverse size $b$.

In the momentum space the standard expression for the transversely polarized photon wave function is [16], [17]:

$$
\begin{aligned}
\psi\left(z, \kappa_{\perp}\right)_{\lambda_{1}, \lambda_{2}} & =e e_{f} \frac{1}{Q^{2}+\frac{\kappa_{\perp}^{2}+m^{2}}{z(1-z)}} \frac{1}{z(1-z)}\left(-\delta(\uparrow \uparrow) m\left(\epsilon^{1}+i \epsilon^{2}\right)+\delta(\downarrow \downarrow)\left(\epsilon^{1}-i \epsilon^{2}\right)\right. \\
& +\delta(\uparrow \downarrow)\left((1-2 z) \vec{\epsilon} \cdot \overrightarrow{\kappa_{\perp}}+i \vec{\epsilon} \times \overrightarrow{\kappa_{\perp}}+\delta(\downarrow \uparrow)\left((1-2 z) \vec{\epsilon} \cdot \overrightarrow{\kappa_{\perp}}-i \vec{\epsilon} \times \overrightarrow{\kappa_{\perp}}\right)\right)
\end{aligned}
$$

Here $e_{f}$ is the electric charge of the quark with flavor $f ; \kappa_{\perp}$ is the transverse momentum of the $q \bar{q}$ pair; $\vec{\epsilon}$ is the polarization vector of the photon. The arrows stand for different helicities of the quarks.

Then we perform a Fourier transform into the impact parameter space: 


$$
\psi(z, b)_{\lambda_{1}, \lambda_{2}}=\frac{1}{(2 \pi)^{2}} \int d^{2} \kappa_{\perp} \psi\left(z, \kappa_{\perp}\right)_{\lambda_{1}, \lambda_{2}} e^{-i \kappa_{\perp} \cdot \overrightarrow{b_{\perp}}}
$$

and study the limit $b \rightarrow 0$. The analysis shows that only spin-flip components of the wave function give the leading contribution at the limit $b \rightarrow 0$ :

$$
\begin{aligned}
\psi(z, b \rightarrow 0)_{\lambda_{1}, \lambda_{2}}=-\frac{i}{2 \pi} e e_{f} & \frac{1}{b}\left(\delta ( \uparrow \downarrow ) \left((1-2 z) \vec{\epsilon} \cdot \overrightarrow{n_{1}}+i \vec{\epsilon} \times \overrightarrow{n_{1}}\right.\right. \\
& \left.+\delta(\downarrow \uparrow)\left((1-2 z) \vec{\epsilon} \cdot \overrightarrow{n_{1}}-i \vec{\epsilon} \times \overrightarrow{n_{1}}\right)\right),
\end{aligned}
$$

where $\overrightarrow{n_{1}}$ is a unit vector in the $x$-direction. At once one can see that the photon wave function is singular at the limit $b \rightarrow 0$. Namely this property leads to a singular behavior of $P_{\gamma}\left(\sigma, Q^{2}\right)$ at small $\sigma$.

Upon averaging over two possible polarizations of the photon (not written explicitly) and two possible helicities of quarks and integrating over $z$ one arrives at the following expression:

$$
\int_{0}^{1} \sum_{\lambda_{1}, \lambda_{2}}\left|\psi(z, b \rightarrow 0)_{\lambda_{1}, \lambda_{2}}\right|^{2} d z=e^{2} e_{f}^{2} \frac{1}{b^{2}} I_{Q C D}^{\prime}\left(Q^{2}\right),
$$

where $I_{Q C D}^{\prime}\left(Q^{2}\right)$ is some numerical factor.

The effective cross section of a $q \bar{q}-$ pair interacting with a nucleon, $\sigma\left(b^{2}\right)$, as a function of the impact parameter $b$, is given by [15]:

$$
\sigma\left(b^{2}\right)=\frac{\pi^{2}}{3} b^{2} x G_{T}\left(x, \tilde{Q}^{2}\right) \alpha_{s}\left(\tilde{Q}^{2}\right)
$$

For $x=10^{-3}$ the effective energy $\tilde{Q}^{2}=9 / b^{2}$ [18]. For small but finite cross sections and, therefore, small $b^{2}$, the combination $x G_{T}\left(\tilde{Q}^{2}\right) \alpha_{s}\left(\tilde{Q}^{2}\right)$ weakly depends on $\tilde{Q}^{2}$. We do not consider this effect in the paper.

Therefore, at small $b^{2}$, one can write:

$$
\frac{d \sigma\left(b^{2}\right)}{d b^{2}}=\frac{\pi^{2}}{3} x G_{T}\left(x, \tilde{Q}^{2}\right) \alpha_{s}\left(\tilde{Q}^{2}\right)
$$

Combining equations (23), (27) and (29) we obtain at the limit $\sigma \rightarrow 0$ :

$$
P_{\gamma}\left(\sigma, Q^{2}\right)=e^{2} \frac{1}{\sigma} I_{Q C D}\left(Q^{2}\right)
$$


where the coefficient $I_{Q C D}\left(Q^{2}\right)$ as a function of $Q^{2}$ is given in Table (III). It was calculated for $\sigma / \sigma_{0}=0.1$ to make a comparison with the similar prediction of GVMD.

One can see from Table (III) that both GVMD model and QCD give answers of the same

order of magnitude. Since the wave function of $\rho$-mesons is $\frac{1}{\sqrt{2}}(|u \bar{u}\rangle-|d \bar{d}\rangle)$, one should use $e_{f}^{2}=1 / 2$ when summing over quark flavors.

Comparing Eqs. (30) and (22), one can see that both GVMD model and QCD predict the singular behavior of $P(\sigma)$ at $\sigma \rightarrow 0: P_{\gamma}(\sigma) \propto 1 / \sigma$. Thus we conclude that the GVMD model exhibits the phenomenon of Color Transparency known from QCD, which means that in the photon, with a noticeable probability, there are hadronic configurations weakly interacting with the target.

\section{HARD DIFFRACTIVE ELECTROPRODUCTION OF PHOTONS}

Another application of GVMD which is important for the interpretation of hard diffractive phenomena at HERA can be found in the diffractive photoproduction in deep inelastic scattering initiated by highly virtual photon: $\gamma^{*}\left(Q^{2}\right)+p \rightarrow \gamma^{*}\left(Q_{f}^{2}\right)+p$.

Within GVDM we found that the ratio of the imaginary parts of the amplitudes for real photon and virtual photon production is:

$$
\begin{gathered}
\frac{\operatorname{Im} A\left(\gamma^{*}\left(Q^{2}\right)+p \rightarrow \gamma\left(Q^{2}=0\right)+p\right)}{\left.\operatorname{Im} A\left(\gamma^{*}\left(Q^{2}\right)+p \rightarrow \gamma^{*}\left(Q^{2}\right)\right)+p\right)}= \\
\left(\sum_{n m} \frac{e}{f_{n}} \frac{M_{n}^{2}}{M_{n}^{2}+Q^{2}} \Sigma_{n m} \frac{e}{f_{m}}\right) / \\
\left(\sum_{n m} \frac{e}{f_{n}} \frac{M_{n}^{2}}{M_{n}^{2}+Q^{2}} \Sigma_{n m} \frac{e}{f_{m}} \frac{M_{m}^{2}}{M_{m}^{2}+Q^{2}}\right) .
\end{gathered}
$$

This ratio is given in Fig. 3. One can see that it grows with $Q^{2}$. Although PQCD predicts that this ratio is approximately $Q^{2}$-independent at small $x$ and estimates it to be $\approx 2$ [19], for a wide range of $Q^{2}$ both answers are rather close. 


\section{CONCLUSIONS}

We have shown that, using the generalized vector meson dominance model, one can reconstruct the distribution function $P_{\gamma}\left(\sigma, Q^{2}\right)$, which gives the probability for the photon to interact with a target with the cross section $\sigma$. The very existence of such a distribution supports the idea that the photon consists of $q \bar{q}$ configurations of a different geometrical size even within nonperturbative QCD regime, therefore they interact with the target with different cross sections.

We have studied the limiting behavior of $P_{\gamma}\left(\sigma, Q^{2}\right)$ at $\sigma \rightarrow 0$ and shown that $P\left(\sigma, Q^{2}\right) \propto$ $1 / \sigma$. That is a very remarkable property which means that there is a sizable probability of finding a small configuration in the photon. Moreover, the relative probability to find such a small configuration in the photon increases when the photon virtuality $Q^{2}$ grows.

We conclude that the GVMD model describes well Bjorken scaling and nuclear shadowing because it takes into account basic coherent QCD phenomena: non-diagonal transitions result in cross section fluctuation of the virtual photon; the specific type of the non-diagonal terms, chosen in line with the parton model, leads to a significant probability of spatially small configurations in the photon wave function and the Color Transparency Phenomenon.

As in the parton model, we found in GVMD that although the contribution of soft QCD in the photon wave function decreases with increase of $Q^{2}$, it gives a comparable contribution into the total cross section and the dominant contribution into moments of $P\left(\sigma, Q^{2}\right)$.

Since the model gives approximate Bjorken scaling of nuclear shadowing, it means that the photon has "hard" and "soft" components, which was suggested by the aligned jet model and affirmed later by QCD. This reveals the duality between the parton language and GVMD description of the photon hadronic structure.

Also within the GVMD model we give predictions for the ratio of the imaginary parts

of the amplitudes of the process $\gamma^{*}\left(Q^{2}\right)+p \rightarrow \gamma^{*}\left(Q_{f}^{2}\right)+p$, which turns out to be consistent with PQCD over a wide range of $Q^{2}$. 


\section{ACKNOWLEDGMENTS}

We thank G. Shaw for very helpful discussions.

We are thankful to D. Schildknecht for pointing out reference [5].

This work was supported by the U.S. Department of Energy grant number DE-FG0293ER-40771 and US-Israeli Bi-National Science Foundation Grant number 9200126. 


\section{REFERENCES}

[1] R.P. Feynman Photon-Hadron Interactions Reading, Mass., W. A.Benjamin, 1972

[2] Bauer, Spital, D.R. Yennie, and Pipkin, Rev. of Mod. Phys. 50, n.2 (1978)

[3] A. Donnachie and G. Shaw, in: Electromagnetic interactions of hadrons, vol.2, 169-194, eds. A. Donnachie and G. Shaw, Plenum, New York (1978).

[4] NMC Collaboration: M. Arneodo at al. Phys. Lett. B364 107 (1995)

[5] H. Fraas, B. Read, and D. Schildknecht, Nucl. Phys. B86, 346 (1975)

[6] G. Shaw, Phys. Rev. D47, R3676 (1993); G. Shaw, Phys. Lett. B228, 125 (1989); P. Ditsas and G. Shaw Nucl. Phys. 113, 246 (1976)

[7] E. Feinberg and I. Pomeranchuk Suppl. Nuovo Cimento III, 652 (1956); M. Good and W. Walker, Phys. Rev. 120, 1857 (1960)

[8] L. Frankfurt, G. Miller, and M. Strikman Ann. Rev. Nucl. Part. Sci 45, 501 (1994)

[9] V. Gribov Sov. Phys. JETP 29, 483 (1969)

[10] H. Miettinen and J. Pumplin, Phys. Rev. D18, 1696 (1978); Phys. Rev. Lett 42, 204 (1979)

[11] B. Kopeliovich and B. Povh Phys. Lett. B3675, 329 (1996)

[12] V. Gribov Sov. Phys. JETP 30, 709 (1970)

[13] J. Bjorken and J. Kogut Phys. Rev. D8, 1341 (1973); J. Bjorken, Proc. Cornell Int. Conf. on Electron and photon interactions (Cornell University Press 1972)

[14] L. Frankfurt and M. Strikman Phys. Rep. 160, 235 (1988)

[15] L. Frankfurt, A. Radyushkin, and M. Strikman, Phys. Rev. D55, 98 (1997)

[16] S. Brodsky, L. Frankfurt, J. Gunion, A. Mueller and M. Strikman, it Phys. Rev. D50, 
$3134(1994)$

[17] S. Brodsky and P. Lepage, Phys. Rev. D22, 2157 (1980)

[18] L. Frankfurt, W. Koepf and M. Strikman Phys. Rev. D54, 3194 (1996)

[19] L. Frankfurt, A. Freund and M. Strikman, preprint hep-ph/9710356 


\section{TABLES}

\begin{tabular}{c|c|c|c|c|c|}
\hline \hline Moments & $Q^{2}=0$ & $Q^{2}=0.5 \mathrm{GeV}^{2}$ & $Q^{2}=1 \mathrm{GeV}^{2}$ & $Q^{2}=2 \mathrm{GeV}^{2}$ & $Q^{2}=5 \mathrm{GeV}^{2}$ \\
\hline$\langle\sigma\rangle$ & 1.1421 & 0.4529 & 0.2755 & 0.1519 & 0.0603 \\
$\left\langle\sigma^{2}\right\rangle$ & 0.8589 & 0.2749 & 0.1452 & 0.0679 & 0.0227 \\
$\left\langle\sigma^{3}\right\rangle$ & 0.7547 & 0.2135 & 0.1043 & 0.0449 & 0.0142 \\
$\left\langle\sigma^{4}\right\rangle$ & 0.7180 & 0.1878 & 0.0883 & 0.0374 & 0.0126 \\
$\left\langle\sigma^{5}\right\rangle$ & 0.7203 & 0.1784 & 0.0829 & 0.0362 & 0.0138 \\
\hline \hline
\end{tabular}

TABLE I. Moments of the distribution $P(\sigma)$ as a function of $Q^{2}$

\begin{tabular}{||c|c|c|}
\hline \hline$Q^{2}=0$ & $I_{V M D}\left(Q^{2}\right)$ & $I_{Q C D}\left(Q^{2}\right)$ \\
\hline 0 & $1.7 \times 10^{-2}$ & $2.8 \times 10^{-2}$ \\
1 & $1.4 \times 10^{-2}$ & $2.5 \times 10^{-2}$ \\
2 & $1.1 \times 10^{-2}$ & $2.3 \times 10^{-2}$ \\
5 & $0.6 \times 10^{-2}$ & $1.9 \times 10^{-2}$ \\
\hline \hline
\end{tabular}

TABLE II. Coefficients $I_{V M D}\left(Q^{2}\right)$ and $I_{Q C D}\left(Q^{2}\right)$ as a function of $Q^{2}$ 


\section{FIGURES}

\section{0 mesons}

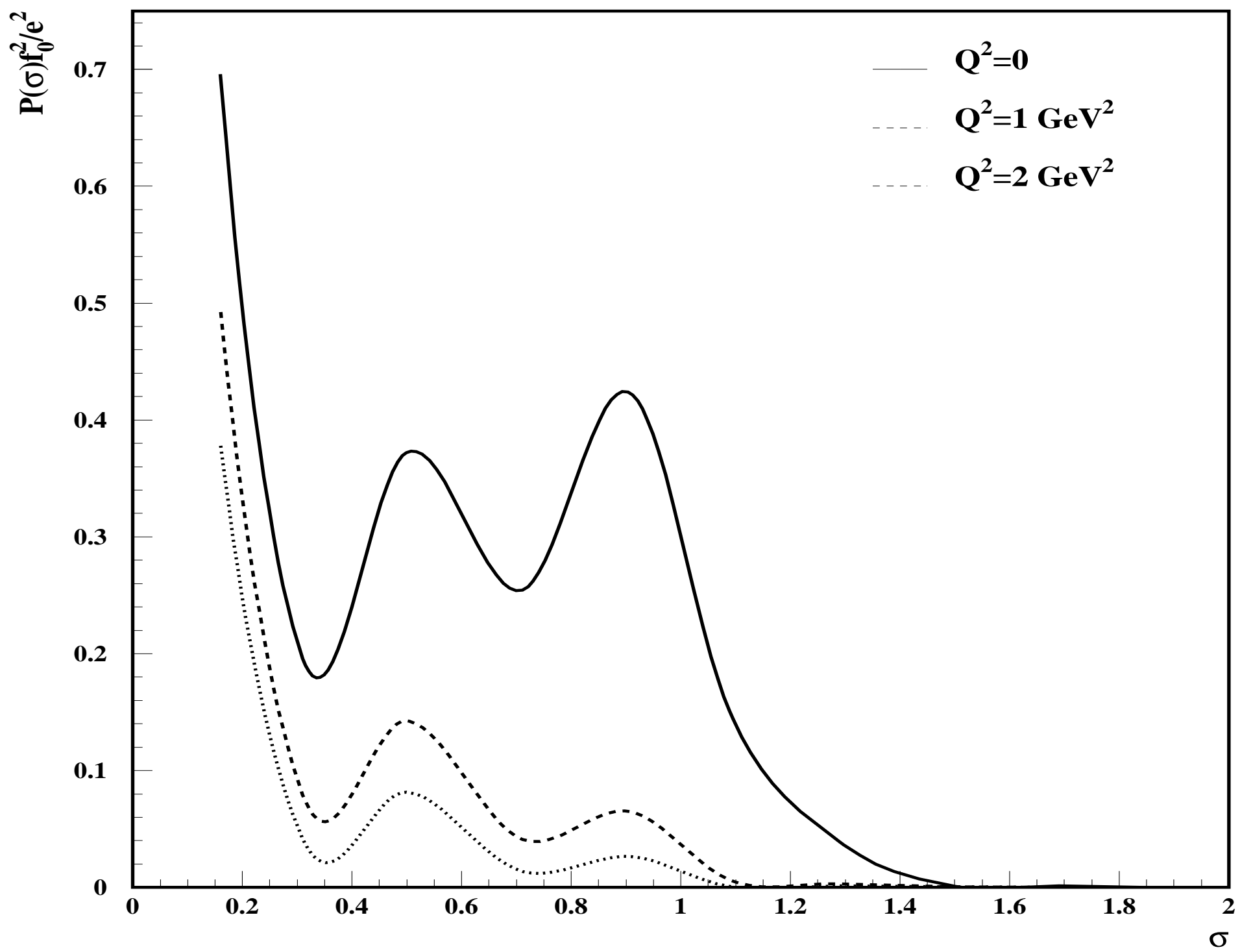

FIG. 1. The distribution $P_{\gamma}\left(\sigma, Q^{2}\right)$ as a function of $Q^{2}$ (we plot $f_{0}^{2} / e^{2} P\left(\sigma, Q^{2}\right)$ ) 


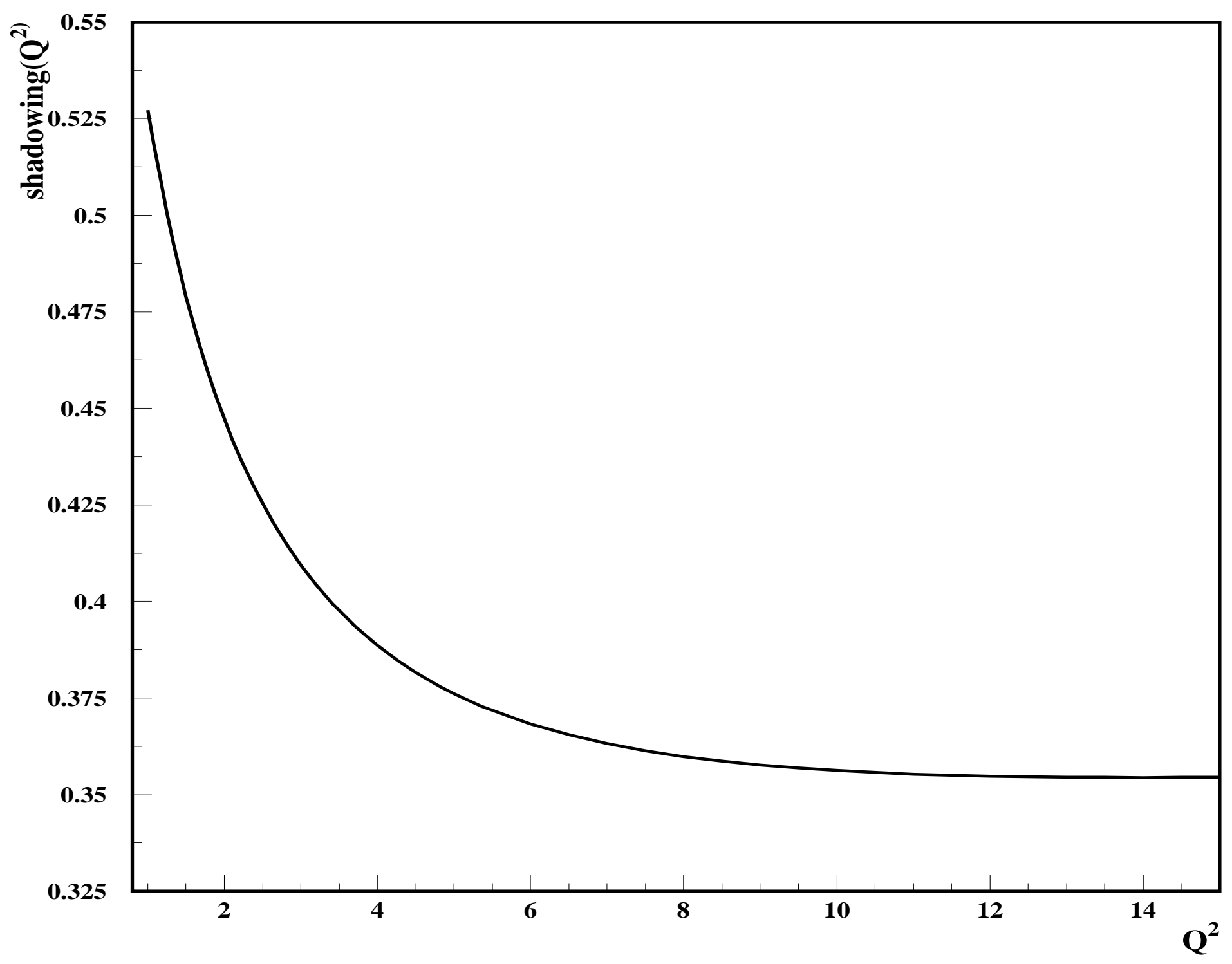

FIG. 2. The shadowing factor shadowing $\left(Q^{2}\right)=\left\langle\sigma^{2}\right\rangle /\left(\langle\sigma\rangle \sigma_{0}\right)$ as a function of $Q^{2}$. $\sigma_{0}$ is the total cross section of interaction of vector mesons with the target. 


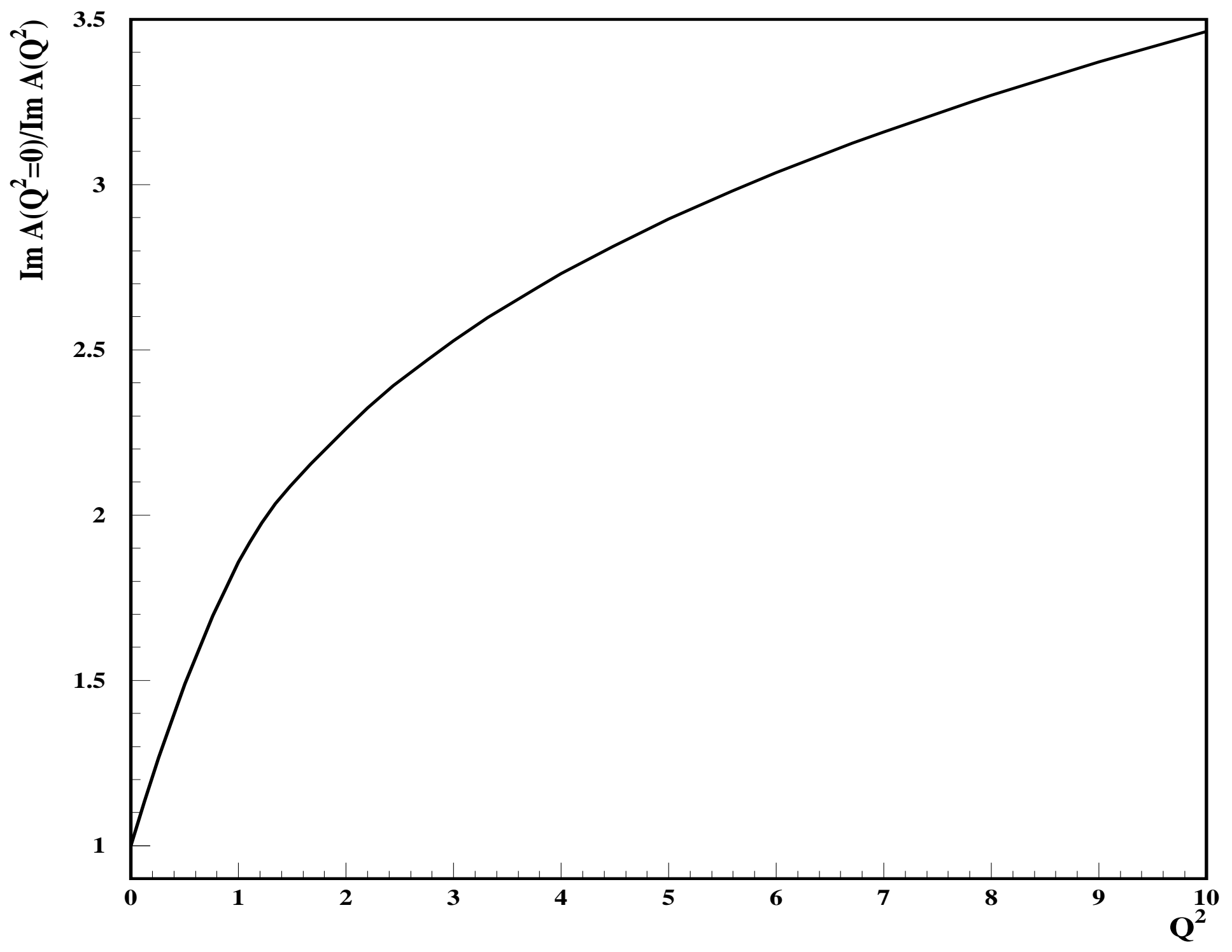

FIG. 3. The fraction of the imaginary parts of the amplitudes of real and virtual photon production as a function of $Q^{2}$. 